\title{
CHARACTER ESTIMATES OF ADJOINT SIMPLE LIE GROUPS
}

\author{
COREY MANACK
}

\begin{abstract}
Call a compact, connected, simple Lie group $G$ adjoint simple if it is also centerless. Let $C \subset G$ be a conjugacy class. We prove the existence of an $n \in \mathbb{N}$, depending on $G$ but not $C$, such that $C^{n}$ contains a neigborhood of the identity. We then prove that a disk $D \subset \mathbb{C}$, with radius less than 1 , contains the image of every normalized character $\chi(e)^{-1} \chi$ of $G$.
\end{abstract}

\section{Introduction, Statement of Results}

We say that a compact, connected Lie group $G$ is simple if its Lie algebra is simple, and $G$ is adjoint simple if $G$ is simple and centerless. Let $\mathfrak{g}=T_{e}(G)$ be the Lie algebra of $G,(\pi, V)$ a finite-dimensional representation of $G, \chi=\operatorname{tr} \pi$ the character of $\pi, e$ the identity element of $G, \operatorname{dim} V=\chi(e)$ the degree of $\pi$. The quantity $\chi(g) / \chi(e) \in \mathbb{C}$ is the normalized character of $V$ at $g \in G$ which is the average of the eigenvalues of $\pi(g)$. By compactness, a normalized character takes values in the closed unit disk in $\mathbb{C}$. When $G$ is adjoint simple, we can say more.

Theorem 1.1. Let $G$ be adjoint simple. Then there exists a real constant $c,-1<$ $c<0$, depending only on $G$, such that the image of any normalized character is contained in the disk tangent to the line $\operatorname{Re} z=c$, and the unit circle.

See Figure 1. The condition that $G$ is adjoint simple is essential. Let $Z(G)$ be the center of $G$. If $G$ is simple then $|Z(G)|$ is finite; if $|Z(G)|>1$, there is a complex irreducible representation $\pi$ and nontrivial $x \in Z(G)$, such that $\pi(x) \neq I$ ([4] pg.

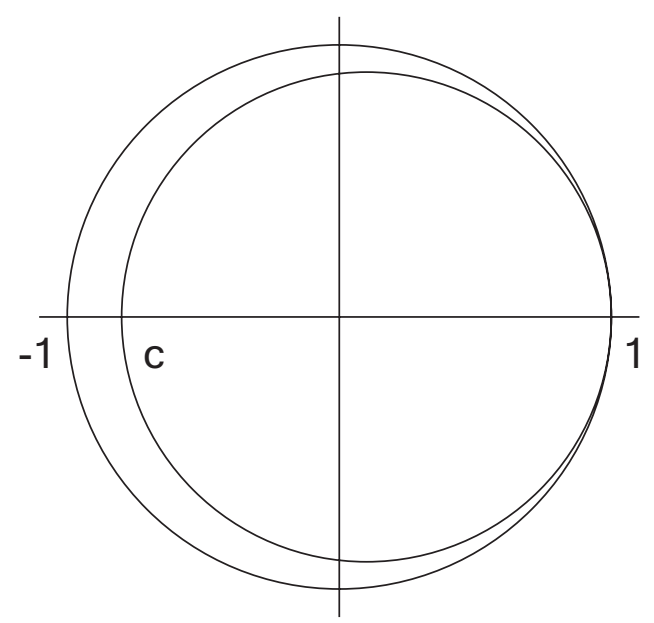

Figure 1. Disk of values for $\frac{\chi(g)}{\chi(e)}$. 
438, Prop, 23.13). As $\pi(x)$ commutes with all $\pi(g)$ and $\pi$ irreducible, $\pi(x)=\lambda I$ by Schur's Lemma. As $G$ is compact, $|\lambda|=1$, and since $x$ has finite order, $\lambda$ is a $k$-th root of unity $\neq 1$. Taking traces, $\lambda=\chi(x) / \chi(e)$ but Theorem 1.1 implies that the image of $\chi(e)^{-1} \chi$ avoids points on the unit circle, except at 1 .

The proof of Theorem 1.1 relies on the following asymptotic structure theorem for products of $n$ elements from a conjugacy class $C \subset G$. Denote by $C^{n}$ the set of $n$-fold products from $C$, i.e.,

$$
C^{n}=\left\{x_{1} x_{2} \cdots x_{n} \mid x_{1}, x_{2}, \ldots, x_{n} \in C\right\} .
$$

Theorem 1.2. Let $G$ be a compact, adjoint simple Lie group. For $n$ sufficiently large, any nontrivial conjugacy class $C \subset G$ has the property that $C^{n}$ contains the identity as an interior point.

We note that conjugacy classes of a compact analytic manifold are regular submanifolds (3) Corollary 2.9.8). If $C$ is nontrivial then $C$ has positive dimension. Intuitively, adjoint simplicity of $G$ implies the dimension of $C^{n}$ increases in $n$ and adjoint simplicity excludes undesirable periodic behavior in $n$. The strategy for proving Theorem 1.2 is standard. Section 2 contains a linearized version of Theorem 1.2 which states, for $n$ sufficiently large and arbitrary $X$ in $\mathfrak{g}$, one can find points $x_{1}, x_{2}, \ldots, x_{n}$ from $G$, such that the function

$$
F\left(g_{1}, \ldots, g_{n}\right)=\operatorname{Ad}\left(g_{1}\right) X+\operatorname{Ad}\left(g_{2}\right) X+\cdots+\operatorname{Ad}\left(g_{n}\right) X
$$

vanishes at $g_{1}=x_{1}, \ldots g_{n}=x_{n}$, and is submersive there. In section 3, we carefully exponentiate (1.1) to prove Theorem[1.2 for conjugacy classes in a punctured neighborhood of $e$. The remaining conjugacy classes follow by a submersion argument and compactness of $G$. Section 4 contains the character estimates. Most of the work is spent proving that the constant $c$ of Theorem 1.1 is greater than -1 . Then $c<0$ from the following observation: for any nontrivial irreducible character $\chi$, orthogonality of $\chi$ to the trivial character implies

$$
\int_{G} \operatorname{Re} \chi(g) d g=0 .
$$

Since $\chi(e)>0, \operatorname{Re} \chi$ must assume a negative value at some non-identity element.

\section{Sums of Adjoint Orbits}

In this section, assume $G$ is simple. We employ tools from convex analysis [2]. Throughout, assume $V$ is a real, finite-dimensional vector space.

Lemma 2.1. Let $V$ be a real vector space and $A$ a compact, convex subset of $V$. Then either

(1) A contains 0 as an interior point, or

(2) $A$ is contained in a closed homogeneous half-space $H$ of $V$, i.e., $v^{*}(A) \geq 0$ for some nonzero dual vector $v^{*} \in V^{*}$.

The proof of Lemma 2.1 can be found in 2.

Theorem 2.2. Let $G$ be simple, $(\pi, V)$ a nontrivial irreducible representation of $G$. If $v \in V$ is nonzero, there exists points $g_{1}, \ldots, g_{n}$ in $G$ for which 0 is contained in the interior of the convex hull of $\pi\left(g_{1}\right) v, \pi\left(g_{2}\right) v, \ldots, \pi\left(g_{n}\right) v$. 
Proof. Let $v \in V \backslash\{0\}$ be given. Suppose towards contradiction that 0 is not in the interior of the convex hull of $\pi(G) v$. Lemma 2.1 says $\pi(G) v$ must lie in a homogeneous half space, which we call $H$. In other words, there exists $v_{0}^{*} \in V$ such that $v_{0}^{*}(\pi(g) v) \geq 0$ for all $g \in G$. As the $\mathbb{R}$-span of $\pi(G) v$ is a nonzero invariant subspace of $V$ and $V$ is irreducible, $\operatorname{span}_{\mathbb{R}} \pi(G) v=V$. Consequently, $\pi(G) v$ is not contained solely in the boundary of $H$, and by continuity of $\pi, v_{0}^{*}(\pi(u) v)>0$ for all $u$ in some open subset $U$ of $G$. Now, define $f \in V^{*}$ to be the covector $f(w)=\int_{G} w^{*}(\pi(g) v) d g$, where $d g$ is the normalized Haar measure on $G$. Since $d g$ is $G$-invariant, so is $f$. Write $f\left(v_{0}\right)$ into the sum of two integrals

$$
f\left(v_{0}\right)=\int_{U} v_{0}^{*}(\pi(g) v) d g+\int_{G \backslash U} v_{0}^{*}(\pi(g) v) d g,
$$

noting that the first integral is positive and the second integral is non-negative. Therefore $f\left(v_{0}\right)>0$, meaning $f$ is a nontrivial $G$-invariant vector of $V^{*}$ which contradicts irreducibility of $V^{*}(\operatorname{dim} V<\infty)$. Thus, 0 can be expressed as a positive convex combination of $\pi\left(g_{1}\right) v, \pi\left(g_{2}\right) v, \ldots, \pi\left(g_{n}\right) v$. As $\operatorname{span}_{\mathbb{R}} \pi(G) v=V$ and $G$ acts transitively on $\pi(G) v$, the vectors $\pi\left(g_{1}\right) v, \pi\left(g_{2}\right) v, \ldots, \pi\left(g_{n}\right) v$ can be chosen so that its convex hull has nonempty interior, augmenting $n$ if necessary.

Definition 1. A smooth map $f: M \rightarrow N$ of manifolds is submersive at $m \in M$ if the differential $d f_{m}$ is surjective; $f$ is called a submersion if it is submersive at every point $m$.

Lemma 2.3. Let $G$ be compact and simple, $(\pi, V)$ an irreducible representation of $G$. For fixed $v \in V$, let $f: G \rightarrow V$ be the map $f(x)=\pi(x) v$. If there are points $x_{1}, x_{2}, \ldots, x_{n}$ such that $f\left(x_{1}\right), \ldots, f\left(x_{n}\right)$ spans $V$, then

$$
d f_{x_{1}} T_{x_{1}}(G)+d f_{x_{2}} T_{x_{2}}(G)+\cdots+d f_{x_{n}} T_{x_{n}}(G)=V .
$$

Proof. Suppose $x:=\left(x_{1}, \ldots, x_{m}\right)$ is a point in $G^{n}$ for which $\left\{\pi\left(x_{i}\right) v\right\}_{i=1}^{n}$ spans $V$. Under the tangent space identifications $T_{x}(V) \cong V$ and

$$
T_{\left(x_{1}, x_{2}, \ldots, x_{n}\right)}\left(G^{n}\right) \cong T_{x_{1}}(G) \times \cdots \times T_{x_{n}}(G),
$$

the statement

$$
d f_{x_{1}} T_{x_{1}}(G)+d f_{x_{2}} T_{x_{2}}(G)+\cdots+d f_{x_{n}} T_{x_{n}}(G)=V
$$

is equivalent to showing that the map

$$
F:\left(g_{1}, g_{2}, \ldots, g_{n}\right) \rightarrow \pi\left(g_{1}\right) v+\pi\left(g_{2}\right) v+\cdots+\pi\left(g_{n}\right) v
$$

is submersive at $x=\left(x_{1}, x_{2}, \ldots, x_{n}\right)$. We show this by taking directional derivatives. For fixed $X \in \mathfrak{g}$ and $n$-tuple of scalars $c=\left(c_{1}, \ldots, c_{n}\right)$, let $\gamma_{X}: \mathbb{R} \times \mathbb{R}^{n} \rightarrow G^{n}$ be the smooth function

$$
\gamma_{X}(t, c)=\left(\exp \left(t c_{1} X\right) x_{1}, \ldots, \exp \left(t c_{n} X\right) x_{n}\right) .
$$

As a function of $t, \gamma_{X}(t, c)$ is a right-translated one parameter subgroup through $x$, with

$$
\gamma_{X}^{\prime}(0, c)=\left(d\left(r_{x_{1}}\right) X, d\left(r_{x_{2}}\right) X, \ldots, d\left(r_{x_{n}}\right) X\right) \in T_{x_{1}}(G) \times \cdots \times T_{x_{n}}(G) .
$$


where $r_{x_{i}}$ is the right multiplication map by $x_{i}$. Explicit calculation shows that the image of $d F_{x}$ contains

$$
\begin{aligned}
d F_{x}\left(d\left(r_{x_{1}}\right) X, d\left(r_{x_{2}}\right) X, \ldots, d\left(r_{x_{n}}\right) X\right) & =\left.\frac{d}{d t}\right|_{t=0} F\left(\gamma_{X}(t, c)\right) \\
& =\left.\frac{d}{d t}\right|_{t=0} \sum_{i=1}^{n} \pi\left(\left(\exp t c_{i} X\right) x_{i}\right) v \\
& =\left.\frac{d}{d t}\right|_{t=0} \sum_{i=1}^{n}\left(\exp t c_{i} d \pi(X)\right) \pi\left(x_{i}\right) v \\
& =d \pi(X) \sum_{i=1}^{n} c_{i} \pi\left(x_{i}\right) v .
\end{aligned}
$$

where the penultimate equality follows from the intertwining property of exp (equation [3.1), and the last equality follows by applying the derivative to each term, factoring $d \pi(X)$ from the sum. As the choice of $X, c$ was arbitrary, the image of $d F_{x}$ contains the subspace

$$
d \pi(\mathfrak{g})\left(\operatorname{span}_{\mathbb{R}}\left\{\pi\left(x_{1}\right) v, \ldots, \pi\left(x_{n}\right) v\right\}\right) .
$$

By assumption, $\operatorname{span}_{\mathbb{R}}\left\{\pi\left(x_{1}\right) v, \ldots, \pi\left(x_{n}\right) v\right\}=V$, and $V$ remains irreducible as a representation of $\mathfrak{g}$, whence $d \pi(\mathfrak{g}) V=V$. The lemma is proved.

Corollary 2.4. Let $G$ be a compact Lie group, $(\pi, V)$ an irreducible representation of $G$. For fixed $v \in V$, let $f: G \rightarrow V$ be the smooth map $f(x)=\pi(x) v$. Suppose the convex hull of $f\left(x_{1}\right), \ldots, f\left(x_{n}\right)$ contains 0 as an interior point. Then

$$
d f_{x_{1}} T_{x_{1}}(G)+d f_{x_{2}} T_{x_{2}}(G)+\cdots+d f_{x_{n}} T_{x_{n}}(G)=V .
$$

Proof. If $f\left(x_{1}\right), f\left(x_{2}\right), \ldots, f\left(x_{n}\right)$ spans a proper subspace of $V$, then the convex hull of

$$
f\left(x_{1}\right), f\left(x_{2}\right), \ldots, f\left(x_{n}\right)
$$

is a subset of $V$ with empty interior. So $f\left(x_{1}\right), f\left(x_{2}\right), \ldots, f\left(x_{n}\right)$ spans $V$, and Lemma 2.3 applies.

Next, we record the version of the Implicit Function Theorem needed for this paper.

Theorem 2.5 (Local Submersion Theorem). Let $M, N$ be smooth manifolds, If $f: M \rightarrow N$ is a submersion at a point $m \in M$, and $f(m)=n$, then there exist local coordinates around $m$ and $n$ such that $f$ is locally equivalent to a projection $\operatorname{map} \mathbb{R}^{k+l} \rightarrow \mathbb{R}^{l}$.

Corollary 2.6. If $f: M \rightarrow N$ is a submersion at $m$ then it is a submersion in a neighborhood of $m$.

Proposition 2.7. Let $n \geq 1$. For positive reals $a_{1}, \ldots, a_{n}$, let $A$ be the ray in $\mathbb{R}^{n}$ emanating from 0 and passing through the point $\left(a_{1}, \ldots, a_{n}\right)$. There exists an infinite sequence of points $x_{1}, x_{2}, \ldots$ in $\mathbb{Z}^{n}$, such that $x_{1}$ is a coordinate vector, $x_{i+1}-x_{i}$ is a coordinate vector, and $x_{i}$ is within distance $\sqrt{2 n}$ of $A$, for all $i$. 
Proof. As $a_{1}, \ldots, a_{n}$ are positive, the function $f: \mathbb{R}^{+} \rightarrow Z^{n}$ given by

$$
f(t)=\left(\left\lfloor t a_{1}\right\rfloor, \ldots,\left\lfloor t a_{n}\right\rfloor\right)
$$

is nondecreasing with respect to the lexicographic ordering $\preceq$. Therefore, the image of $f$ can be listed as a sequence $y_{0}, y_{1}, y_{2}, \ldots$ of points in $\mathbb{Z}^{n}$ with the properties $y_{0}=0, y_{i} \prec y_{i+1}$ for all $i \in \mathbb{N}$. Hence, the difference $y_{i+1}-y_{i}$ is $\sum_{k=1}^{n} \delta_{k} e_{k}$, where $\delta_{k}=0$ or 1 and $\delta_{k}=1$ for at least one $k$. We may therefore construct a sequence of lattice points $x_{1}, x_{2}, \ldots$, containing $y_{1}, y_{2}, \ldots$ as a subsequence, such that $x_{1}$ is a coordinate vector, and $x_{i+1}-x_{i}$ a coordinate vector. The definition of the floor function implies, for all nonnegative $t$,

$$
\sup _{i}\left|\left\lfloor t a_{i}\right\rfloor-t a_{i}\right|^{2} \leq 1
$$

For those $t$ such that $f(t)=y_{k}$, we have, by triangle inequality,

$$
\left|y_{k}-t\left(a_{1}, \ldots, a_{n}\right)\right|^{2} \leq \sup _{i} n\left|\left\lfloor t a_{i}\right\rfloor-t a_{i}\right|^{2} n \leq n
$$

and

$$
\left|y_{k+1}-t\left(a_{1}, \ldots, a_{n}\right)\right|^{2} \leq 2 n .
$$

By construction, for any $x_{l}$ there exists $y_{k}$ so that $y_{k} \preceq x_{l} \prec y_{k+1}$; it follows from the last estimate that

$$
\inf _{t \in \mathbb{R}^{+}}\left|x_{l}-t\left(a_{1}, \ldots, a_{n}\right)\right|^{2} \leq 2 n
$$

The sequence $x_{1}, x_{2} \ldots$ satisfies the lemma.

Lemma 2.8. Let $S=\left\{v_{1}, \ldots v_{n}\right\}$ be a set of vectors in $V$ and $a_{1}, \ldots, a_{n}$ positive real numbers such that $\sum_{i=1}^{n} a_{i} v_{i}=0$. Then there exists $R>0$, and a sequence $w_{1}, w_{2}, \ldots$ of vectors from $S$ such that all partial sums $\sum_{i=1}^{n} w_{i}$ are bounded by $R$.

Proof. Let $A$ be the ray in $\mathbb{R}^{n}$ anchored at 0 and passing through $a=\left(a_{1}, \ldots, a_{n}\right)$. By Proposition 2.7, there exists a sequence of points $x_{1}, x_{2}, \ldots$ from $\mathbb{Z}^{n}$, such that $x_{i}$ is within distance $\sqrt{2 n}$ of $A$, and consecutive terms differ by a coordinate vector. Let $t a$ be a point on the ray whose distance to $x_{k}$ is at most $\sqrt{2 n}$; if we write $x_{k}$ as $\left(x_{k 1}, x_{k 2} \ldots, x_{k n}\right)$, then by comparing coordinates,

$$
\sup _{i}\left|x_{k i}-t a_{i}\right| \leq\left|x_{k}-t a\right|<\sqrt{2 n} .
$$

By assumption, $t a_{1} v_{1}+\cdots+t a_{n} v_{n}=0$, yielding

$$
\begin{aligned}
\left|x_{k 1} v_{1}+\ldots+x_{k n} v_{n}\right| & =\left|x_{k 1} v_{1}+\cdots+x_{k n} v_{n}-\left(t a_{1} v_{1}+\cdots+t a_{n} v_{n}\right)\right| \\
& \leq\left|x_{k 1}-t a_{1}\right|\left|v_{1}\right|+\cdots+\left|x_{k n}-t a_{n}\right|\left|v_{n}\right| \\
& \leq n \sqrt{2 n} \sup _{j}\left|v_{j}\right| .
\end{aligned}
$$

Let $R=n \sqrt{2 n} \sup _{j}\left|v_{j}\right|$ and define $f: \mathbb{Z}^{n} \rightarrow V$ to be the linear map

$$
f\left(b_{1}, \ldots, b_{n}\right)=b_{1} v_{1}+b_{2} v_{2}+\ldots+b_{n} v_{n} .
$$

Notice that $f$ maps the set of coordinate vectors of $\mathbb{R}^{n}$ onto $S$. By the construction in Proposition 2.7 $f\left(x_{1}\right) \in S$, and since $x_{i+1}-x_{i}$ is a coordinate vector, $f\left(x_{i+1}-\right.$ $\left.x_{i}\right) \in S$. Explicitly, the sequence

$$
f\left(x_{1}\right), f\left(x_{2}-x_{1}\right), f\left(x_{3}-x_{2}\right), \ldots
$$

has $k$-th partial sum $f\left(x_{k}\right)$, which was just shown to satisfy $\left|f\left(x_{k}\right)\right| \leq R$. 
Theorem 2.9. Let $V$ be a finite dimensional real vector space, $M$ a smooth manifold, $f: M \rightarrow V$ a smooth function, $x_{1}, \ldots, x_{m}$ points of $M$ such that 0 lies in the interior of the convex hull of $f\left(x_{1}\right), \ldots, f\left(x_{m}\right)$, and

$$
V=d f_{x_{1}} T_{x_{1}}(M)+\cdots+d f_{x_{m}} T_{x_{m}}(M) .
$$

Then, for all $n$ sufficiently large, there exist $z_{1}, \ldots, z_{n} \in M$ such that

$$
\sum_{i=1}^{n} f\left(z_{i}\right)=0
$$

and

$$
V=d f_{z_{1}} T_{z_{1}}(M)+\cdots+d f_{z_{n}} T_{z_{n}}(M) .
$$

Proof. Since 0 lies in the interior of the convex hull of $f\left(x_{1}\right), \ldots, f\left(x_{m}\right)$, for $\epsilon>0$ sufficiently small, we can find convex coefficients $a_{1}, a_{2}, \ldots, a_{m}$ such that

$$
a_{1} f\left(x_{1}\right)+a_{2} f\left(x_{2}\right)+\cdots+a_{m} f\left(x_{m}\right)=-\epsilon\left(f\left(x_{1}\right)+\cdots+f\left(x_{m}\right)\right) .
$$

Solving and renormalizing, $f\left(x_{1}\right), \ldots, f\left(x_{m}\right)$ satisfy

$$
a_{1} f\left(x_{1}\right)+a_{2} f\left(x_{2}\right)+\cdots+a_{m} f\left(x_{m}\right)=0
$$

and each convex coefficient $a_{1}, a_{2}, \ldots, a_{m}$ in (2.4) is positive. Keeping $m$ fixed but allowing $k \in \mathbb{N}$ to vary, write $a \in \mathbb{R}^{m}$ as $a=\left(a_{1}, \ldots, a_{m}\right), x \in M^{m}$ as $x=\left(x_{1}, \ldots, x_{m}\right), t \in \mathbb{R}^{k}$ as $t=\left(t_{1}, \ldots, t_{k}\right), y \in M^{k}$ as $y=\left(y_{1}, \ldots, y_{k}\right)$, and let $\mathbb{1}_{k}=(1, \ldots, 1)\left(k\right.$ times). Define the family of smooth maps $F_{1}, F_{2}, \ldots$ to be

$$
F_{k}(t, y)=\sum_{i=1}^{k} t_{i} f\left(y_{i}\right),
$$

and let $F_{k}^{t}: M^{k} \rightarrow V$ be the restriction of $F_{k}$ to the slice $\{t\} \times M^{k}$. If every component of $t$ is nonzero, the image of the differential of $F_{k}^{t}$ at $y$ is the subspace

$$
d f_{y_{1}} T_{y_{1}}(M)+\cdots+d f_{y_{k}} T_{y_{k}}(M) .
$$

So the theorem is proved once we produce an $N \in \mathbb{N}$ such that, for all $n \geq N$ some point $z=\left(z_{1}, \ldots, z_{n}\right)$ in $M^{n}$ satisfies $F_{n}^{\mathbb{1}_{n}}(z)=0$ and $F_{n}^{\mathbb{1}_{n}}$ is submersive at $z$, because the coordinates of such a $z$ would satisfy properties (2.2) and (2.3) simultaneously. At this point, we know $F_{m}^{a}(x)=F_{m}(a, x)=0$ and $F_{m}^{a}$ is submersive at $x$. The Implicit Function Theorem grants a $\delta>0$ and a smooth function

$$
g: a+(-\delta, \delta)^{m} \rightarrow M^{m}
$$

satisfying $g(a)=x, F_{m}^{t}(g(t))=F_{m}(t, g(t))=0$ for $t$ satisfying $\sup _{i}\left|t_{i}-a_{i}\right|<$ $\delta$. By Corollary 2.6 and our spanning assumption (2.1), $F_{m}^{t}$ is a submersion at all points in a neighborhood $U$ of $x$, so long as each component of $t$ is nonzero. Choosing $\delta$ small enough so that each $t \in a+(-\delta, \delta)^{m}$ has positive coordinates and $g\left(a+(-\delta, \delta)^{m}\right) \subset U$, we may assume that $F_{m}^{t}$ is a submersion at $y=g(t)$ for all $t \in a+(-\delta, \delta)^{m}$. In particular, there exists an $m$-tuple of positive rationals

$$
\frac{p}{q}:=\left(\frac{p_{1}}{q_{1}}, \frac{p_{2}}{q_{2}}, \ldots, \frac{p_{m}}{q_{m}}\right)
$$

such that $p_{i} / q_{i}$ falls within $\delta$ of $a_{i}$. It follows that $F_{m}^{p / q}$ vanishes at the point

$$
c:=\left(c_{1}, \ldots, c_{m}\right):=g\left(\frac{p_{1}}{q_{1}}, \frac{p_{2}}{q_{2}}, \ldots, \frac{p_{m}}{q_{m}}\right) \in X^{m}
$$


and $F_{m}^{p / q}$ is submersive at $c$. Denote by $\Delta^{k}$ the diagonal submanifold of $M^{k}$; if we let

$$
n=p_{1} q_{2} \cdots q_{m}+q_{1} p_{2} \cdots q_{m}+\cdots+q_{1} q_{2} \cdots p_{m}
$$

then notice that the function $q_{1} q_{2} \cdots q_{m} F_{m}^{p / q}$ is simply the restriction of $F_{n}^{\mathbb{1}_{n}}$ to the product of diagonal manifolds

$$
\Delta^{p_{1} q_{2} \cdots q_{m}} \times \Delta^{q_{1} p_{2} \cdots q_{m}} \times \cdots \times \Delta^{q_{1} q_{2} \cdots p_{m}} \subset M^{n} .
$$

Therefore, the image of the differential of $q_{1} q_{2} \cdots q_{m} F_{m}^{p / q}$ at $c$ is a subspace of the image of the differential of $F_{n}^{\mathbb{1}_{n}}$ at

$$
z=\left(z_{1}, \ldots, z_{n}\right):=(\underbrace{c_{1}, \ldots, c_{1}}_{p_{1} q_{2} \cdots q_{m}}, \underbrace{c_{2}, \ldots, c_{2}}_{q_{1} p_{2} \cdots q_{m}}, \ldots, \underbrace{c_{m}, \ldots, c_{m}}_{q_{1} q_{2} \cdots p_{m}}) .
$$

Since $q_{1} q_{2} \cdots q_{m} F_{m}^{p / q}(c)=0$ and $q_{1} q_{2} \cdots q_{m} F_{m}^{p / q}$ is submersive at $c$, we've shown the existence of an $n$, given by equation (2.5) and $n$-tuple $z=\left(z_{1}, \ldots, z_{n}\right)$, defined in (2.6), such that $F_{n}^{\mathbb{1}_{n}}(z)=0$ and $F_{n}^{\mathbb{1}_{n}}$ is submersive at $z$. The theorem would follow once (2.2) and (2.3) are satisfied for all integers beyond a sufficiently large multiple of $n$. Consider $F_{n}^{\mathbb{1}}{ }^{n}$ near $z$. By Theorem 2.6. there exists an open set $Z \subset M^{n}$ containing $z$ and an open ball $B_{r}(0) \subset V$ such that $F_{n}^{\mathbb{1}_{n}}$ is a submersion on $Z$ and $F_{n}^{\mathbb{1}_{n}}(Z)=B_{r}(0)$. Notice further, for all $k, l \in \mathbb{N}$, the function $F_{l n+k}^{\mathbb{1}_{l n+k}}$ remains a submersion at all points in $Z^{l} \times X^{k}$. By linearity,

$$
F_{l n+k}^{\mathbb{1}_{l n+k}}\left(Z^{l} \times\{y\}\right)=B_{l r}(0)+\sum_{i=1}^{k} f\left(y_{i}\right) .
$$

Now, recalling that 0 lies in the interior of the convex hull of $f\left(x_{1}\right), f\left(x_{2}\right), \ldots, f\left(x_{m}\right)$, by Lemma 2.8, there is a bound $R>0$ and sequence of vectors $f\left(x_{i_{1}}\right), f\left(x_{i_{2}}\right) \ldots$, where $x_{i_{j}} \in\left\{x_{1}, x_{2}, \ldots, x_{m}\right\}$, such that the sequence of vectors

$$
s_{k}:=\sum_{j=1}^{k} f\left(x_{i_{j}}\right)=F_{k}\left(\mathbb{1}_{k}, x_{i_{1}}, \ldots, x_{i_{k}}\right)
$$

satisfy $s_{k} \in B_{R}(0)$ for all $k \in \mathbb{N}$. Consequently, if $l \in \mathbb{N}$ is chosen to satisfy $l r \geq R$, then the translated ball $B_{l r}(0)+s_{k} \subset V$ contains 0 , for all $k \in \mathbb{N}$. By (2.7), there exists a point $z^{*} \in Z^{l} \times\left\{x_{1}, \ldots, x_{m}\right\}^{k}$ such that $F_{l n+k}^{\mathbb{1}_{l n+k}}\left(z^{*}\right)=0$ and $F_{l n+k}^{\mathbb{1}_{l n+k}}$ is submersive at $z^{*}$. Therefore, if $n^{\prime} \geq \ln$, there exists $z^{*} \in M^{n^{\prime}}$ whose coordinates satisfy (2.2) and (2.3). The Theorem is proved.

Let $G$ be simple. For fixed $X \in \mathfrak{g}$, let $f(x)=\operatorname{Ad}(x) X$. Viewing $\mathfrak{g}$ as a real vector space on which Ad acts irreducibly ([4], page 434), Lemmas 2.2 2.4 specialize to yield 0 as expressed by a sufficiently large convex combination of vectors $\operatorname{Ad}\left(x_{1}\right) X, \ldots, \operatorname{Ad}\left(x_{n}\right) X$, and the function

$$
\left(g_{1}, \ldots, g_{n}\right) \rightarrow \operatorname{Ad}\left(g_{1}\right) X+\cdots+\operatorname{Ad}\left(g_{n}\right) X
$$

is submersive at $\left(x_{1}, \ldots, x_{n}\right)$. Theorem 2.9 shows that the convex coefficients $a_{1}, \ldots, a_{n}$ can be replaced by sufficiently large positve integer coefficients $b_{1} \ldots, b_{n}$, retaining the vanishing and submersivity properties of Corollary 2.4. In other words, for sufficiently large $n$, the sum of $n$ copies of $\operatorname{Ad}(G) X$ contains a neighborhood of 
0 . Notice that $n$ may depend on $X$. We lift this dependency, at the group level, in the next section.

\section{Powers of a Conjugacy Class}

We record the three properties of the exponential map exp: $\mathfrak{g} \rightarrow G$ used in this section [3]: $t \rightarrow \exp t X$ is a homomorphism from $\mathbb{R}$ to $G$, exp is a local diffeomorphism near $0 \in \mathfrak{g}$ whose local inverse we denote by $\log$, and if $(\pi, V)$ is any representation of $G$, exp intertwines the $\pi$ with its differential $d \pi$, i.e.,

$$
\pi \circ \exp =\exp \circ d \pi
$$

for all $X \in \mathfrak{g}$. The fourth property, Theorem 3.1 is the Baker-Campbell-Hausdorff $(\mathrm{BCH})$ formula, which expresses the product of $n$ elements near $e$ in terms of their exponentials. The next results are developed in [3, Section 2.15] for $n=2$ and can be extended to arbitrary $n$ by induction.

Theorem 3.1 ([3], Theorem 2.12.4, Theorem 2.15.4, equation (2.15.5)). Let $G$ be a Lie group, $\mathfrak{g}$ the Lie algebra of $G, S(\mathfrak{g})$ the unit sphere in $\mathfrak{g}$ with respect to the Killing form, $B(\mathfrak{g})$ the unit ball, $n \geq 1$, and $X_{1}, \ldots, X_{n} \in \mathfrak{g}$. There exists a unique smooth function $r_{n}: \mathfrak{g}^{n} \rightarrow \mathfrak{g}$, such that, for $|t|$ sufficiently small,

$$
\exp t X_{1} \cdots \exp t X_{n}=\exp \left(t\left(X_{1}+\cdots+X_{n}\right)+r_{n}\left(t X_{1}, \ldots, t X_{n}\right)\right) .
$$

Moreover, for $l=1,2$ consider the two functions

$$
R_{n}\left(t, X_{1}, \ldots, X_{n}\right)=\left\{\begin{array}{cc}
t^{-l} r_{n}\left(t X_{1}, \ldots, t X_{n}\right), & t \neq 0, \\
0 & t=0
\end{array}\right.
$$

$R_{n}$ is smooth when $l=1$, and $R_{n}$ is bounded (with respect to the Killing form) when $l=2,|t|$ sufficiently small and $X_{1}, \ldots, X_{n} \in S$.

Remark 1. The remainder function $r_{n}$ is a power series in the commutators of $X_{1}, \ldots, X_{n}$, given in closed form by Dynkin's formula [3, remark 3]. All we need are the properties of $r_{n}$ stated in equation (3.2).

Define $w_{n}: G^{n} \times \mathfrak{g} \rightarrow G$ to be the word map

$$
w_{n}\left(g_{1}, g_{2}, \ldots, g_{n}, X\right)=g_{1} \exp X g_{1}^{-1} g_{2} \exp X g_{2}^{-1} \cdots g_{n} \exp X g_{n}^{-1} \text {. }
$$

The image of $w_{n}$ is the set of $n$-fold products of the conjugacy class through $\exp X$; we would like to examine the image of $\log w_{n}$. To do so requires that the image of $w_{n}$ be contained in the domain of log.

Proposition 3.2. Let $G$ be a compact connected Lie group, $S=S(\mathfrak{g})$ the unit sphere in $\mathfrak{g}$ with respect to the Killing form $B=B(\mathfrak{g})$ the unit ball. For all $n>0$, there exists $\delta$ sufficiently small and $\mu>0$ such that for any $1 \leq k \leq n$ and $0<t<\delta$ the product of $k$ elements from $\exp (t S)$ is contained in $\exp (t \mu B)$.

Proof. Let $n>0$ be given, $R$ the the injectivity radius of $G, \delta$ small enough so that $\exp (\delta B)^{k} \subset \exp R B$, for all $1 \leq k \leq n$. Let $1 \leq k \leq n, 0<t<\delta$, $X_{1}, \ldots, X_{k} \in S(\mathfrak{g})$; by BCH,

$$
\log \left(\exp \left(t X_{1}\right) \exp \left(t X_{2}\right) \cdots \exp \left(t X_{k}\right)\right)=t X_{1}+\cdots+t X_{k}+r_{k}\left(t X_{1}, \ldots, t X_{k}\right),
$$

and by equation (3.2) (case $l=2$ ), there exists a bound $m_{k}>0$ such that $\left|r_{k}\left(t X_{1}, \ldots, t X_{k}\right)\right|<t^{2} m_{k}$ for all $X_{1} \ldots, X_{k} \in S(\mathfrak{g})$. Consequently,

$$
\left|\log \left(\exp \left(t X_{1}\right) \exp \left(t X_{2}\right) \cdots \exp \left(t X_{k}\right)\right)\right| \leq t k+t^{2} m_{k} .
$$


Choosing $\mu=\max \left\{k+\delta m_{k} \mid 1 \leq k \leq n\right\}$, observe

$$
t k+t^{2} m_{k}<t\left(k+\delta m_{k}\right) \leq t \mu
$$

implies

$$
\log \left(\exp \left(t X_{1}\right) \exp \left(t X_{2}\right) \cdots \exp \left(t X_{k}\right)\right) \in t \mu B
$$

for all $1 \leq k \leq n, 0<t<\delta, X_{1}, \ldots, X_{k} \in S$. The proof follows by exponentiation.

The next lemma will provide sufficient control over the $r_{n}$ term of the $\mathrm{BCH}$ formula to prove Theorem 3.4 for sufficienly large multiples of conjugacy classes $C$ near $e$. Then Proposition 3.2 is used to fill in intermediate powers of $C$. Abusing notation, write $g(u, V)$ for the image $g(\{u\} \times V)$.

Lemma 3.3. Let $M, N, P$ be smooth manifolds, $g: M \times N \rightarrow P$ a smooth function, $g^{a}$ the restriction of $g$ to the slice $\{a\} \times N$. Suppose $g\left(a_{0}, b_{0}\right)=c_{0}$ and $g^{a_{0}}$ is a submersion at $b_{0}$. Then there is an open set $U \subset M$ containing $a_{0}$, an open set $V \subset N$ containing $b_{0}$, an open set $W \subset P$ containing $c_{0}$, such that $W \subset g(u, V)$ for all $u \in U$.

Proof. We construct, from $g$, a smooth function so as to apply the local submersion theorem. Define $f: M \times N \rightarrow M \times P$ to be

$$
f(m, n)=(m, g(m, n)) .
$$

As $d f=i d \times d g$, the image of $d g$ at $\left(a_{0}, b_{0}\right)$ includes the image of $d\left(g^{a_{0}}\right)$ at $b_{0}$ and $g^{a_{0}}$ submersive at $b_{0}, f$ is submersive at $\left(a_{0}, b_{0}\right)$. By Theorem [2.5, $f$ maps some open neighborhood $U \times V$ of $\left(a_{0}, b_{0}\right)$ onto an open neighborhood $Q$ of $\left(a_{0}, c_{0}\right)$. $Q$ contains a neighborhood of the form $U_{1} \times W$. Comparing second coordinates, we see $g(u, V)$ contains $W$ for all $u \in U_{1}$.

Definition 2. Let $G$ be a Lie group, $C$ a conjugacy class of $G, S$ a subset of $G$. We say that $C$ is represented in $S$ if $S \cap C \neq \emptyset$, i.e. $C$ is represented by some $x \in S$.

The next Theorem proves Theorem 1.2 for conjugacy classes sufficiently close to $e$.

Theorem 3.4. Let $G$ be an adjoint simple Lie group. There exists a punctured neighborhood $U_{e}^{*}$ of the identity of $G$, such that, if $C$ is a conjugacy class represented in $U_{e}^{*}$, then, for all $n$ sufficiently large, $C^{n}$ contains $e$ as an interior point.

Proof. Denote by $S$ the unit sphere in $\mathfrak{g}$ with respect to the Killing form, $B$ the unit ball in $\mathfrak{g}$. As $G$ is adjoint simple, $\mathfrak{g}$ is a real vector space on which the adjoint representation of $G$ acts irreducibly. For fixed $X \in S$, let $f(g)=\operatorname{Ad}(g) X$; Theorem 2.2. specialized to $\pi=\operatorname{Ad}, v=X$, asserts the existence of points $x_{1}, \ldots, x_{m} \in G$, for which zero lies in the interior of the convex hull of

$$
f\left(x_{1}\right), f\left(x_{2}\right) \ldots, f\left(x_{m}\right),
$$

so that Corollary 2.4 implies

$$
d f_{x_{1}} T_{x_{1}}(G)+\cdots+d f_{x_{m}} T_{x_{m}}(G)=V .
$$

Write $g=\left(g_{1}, \ldots, g_{n}\right)$ and let $F_{1}, F_{2} \ldots$ be the family of maps where $F_{n}: S \times G^{n} \rightarrow$ $\mathfrak{g}$ is given by

$$
F_{n}(Y, g)=\operatorname{Ad}\left(g_{1}\right) Y+\operatorname{Ad}\left(g_{2}\right) Y+\cdots+\operatorname{Ad}\left(g_{n}\right) Y
$$


$F_{n}^{Y}$ the restriction of $F_{n}$ to the slice $\{Y\} \times G^{n}$. As $f$ satisfies the convexity and submersivity conditions in the supposition of Theorem 2.9, then for $n$ sufficiently large, there is a point $z=z=\left(z_{1}, \ldots, z_{n}\right) \in G^{n}$ satisfying

$$
F_{n}(X, z)=\operatorname{Ad}\left(z_{1}\right) X+\operatorname{Ad}\left(z_{2}\right) X+\cdots+\operatorname{Ad}\left(z_{n}\right) X=0,
$$

and $F_{n}^{X}$ is submersive at $x$. Fix one such $n, z$ pair, and let $R>0$ is the injectivity radius of $G, \delta$ small enough so that $\exp (\delta B)^{k} \subset \exp R B$, for all $1 \leq k \leq n$. Since spheres in $\mathfrak{g}$ with respect to the Killing form are Ad-invariant, $\exp (\delta \operatorname{Ad}(G) B)^{k} \subset$ $\exp (R B)$, for all $1 \leq k \leq n$. Recall the remainder function $r_{n}$ of the $\mathrm{BCH}$ formula (3.1); consider the function $h:(-\delta, \delta) \times S \times G^{n} \rightarrow \mathfrak{g}$ is given by

$$
h(t, Y, g)=\left\{\begin{array}{cc}
t^{-1}\left(F_{n}(t Y, g)+r_{n}\left(t \operatorname{Ad}\left(g_{1}\right) Y, \ldots, t \operatorname{Ad}\left(g_{n}\right) Y\right)\right), & t \neq 0, \\
F_{n}(Y, g) & t=0
\end{array}\right.
$$

By equation (3.2) (case $l=1$ ), $h$ is smooth for all $|t|$ sufficiently small. Further, $h(0, X, z)=F_{n}^{X}(z)=0$. Apply Lemma 3.3 to the special case $M=(-\delta, \delta) \times S$, $a_{0}=(0, X), N=G^{n}, b_{0}=x, P=\mathfrak{g}, c_{0}=0$ to assert the existence of $\epsilon>0$, an open set $U_{X} \subset S$ containing $X$, an open set $V \subset G^{n}$ containing $z$, and an open ball $\rho B \subset \mathfrak{g}$, such that

$$
\rho B \subset h(t, Y, V),
$$

for all $Y \in U_{X}, t \in(-\epsilon, \epsilon)$. Thus, for $t \in(0, \epsilon)$ and $Y \in U_{X}, \mathrm{BCH}$ and the intertwining property of equation (3.1) shows

$$
\begin{aligned}
& \exp t h(t, Y, g) \\
= & \exp \left(t \operatorname{Ad}\left(g_{1}\right) Y\right) \exp \left(t \operatorname{Ad}\left(g_{2}\right) Y\right) \cdots \exp \left(t \operatorname{Ad}\left(g_{n}\right) Y\right) \\
= & \left.g_{1} \exp (t Y) g_{1}^{-1} g_{2} \exp (t Y) g_{2}^{-1} \cdots g_{n} \exp (t Y)\right) g_{n}^{-1} \\
= & w_{n}\left(g_{1}, \ldots, g_{n}, t Y\right)
\end{aligned}
$$

Notice, if $C$ is the conjugacy class $C=\exp (t \operatorname{Ad}(G) Y)$, then $\exp \operatorname{th}\left(t, Y, G^{n}\right)=C^{n}$. Consequently, for all $t \in(0, \epsilon), Y \in U_{X} \subset S$, if $C=\exp (t \operatorname{Ad}(G) Y)$, then $C^{n}$ contains the open neighborhood $\exp t \rho B$ of $e$. It follows from

$$
\exp (b B) \subset \exp (B)^{b}
$$

that $C^{b n}$ contains $\exp t b \rho B$ for all positive integers $b$. Since $t<\epsilon<\delta$ and $\delta$ satisfies the supposition of Proposition 3.2, there exists $\mu>0$ such that $C^{i} \subset \exp t \mu B$ for all $1 \leq i \leq n$. Selecting $b_{0}$ to satisfy $b_{0} \rho \geq \mu$, it follows that $e \in \operatorname{Int}\left(C^{i}\right)$ for all $i \geq b_{0} n$. Thus, for any conjugacy class $C$ represented in the exponentiated cone $\left\{\exp (t Y) \mid t \in(0, \epsilon), Y \in U_{X}\right\}, e \in \operatorname{Int}\left(C^{i}\right)$ for all $i \geq b_{0} n$. Repeating the above argument at each point $X \in S$ yields a cover of $S$ by neighborhoods $U_{X}$ satisfying equation 3.4. By compactness of $S$, we may produce the desired punctured neigborhood of $e$ as a finite union of exponentiated cones $\mathcal{C}_{1}, \ldots, \mathcal{C}_{l}$, taking the maximum necessary exponent over this union.

Proof of Theorem 1.2. Let $G$ be adjoint simple; by Theorem 3.4 there exists a punctured open neighborhood $U=U_{e}^{*}$ of the identity, and sufficiently large $m$ such that, for any conjugacy class $C$ represented in $U_{e}^{*}, C^{m}$ contains $e$ as an interior point. We will complete the proof by proving the following statement: for $N$ sufficiently large, $C^{N}=G$ for any conjugacy class $C$ represented in $G \backslash U_{e}$. 
For $C \neq\{e\}$ and points $x_{1}, x_{2}, \ldots, x_{n} \in C$, consider the map $u_{n}: G^{n} \rightarrow G$ given by

$$
u_{n}\left(g_{1}, \ldots, g_{n}\right)=g_{1} x_{1} g_{1}^{-1} g_{2} x_{2} g_{2}^{-1} \cdots g_{n} x_{n} g_{n}^{-1} x_{n}^{-1} \cdots x_{1}^{-1} .
$$

The image of the differential of $u_{n}$ at $(e, \ldots, e)$ is the subspace

$$
\begin{aligned}
L_{n}\left(x_{1}, \ldots, x_{n}\right):=\left(1-\operatorname{Ad}\left(x_{1}\right)\right)(\mathfrak{g}) & +\operatorname{Ad}\left(x_{1}\right)\left(1-\operatorname{Ad}\left(x_{2}\right)\right)(\mathfrak{g})+\cdots \\
& +\operatorname{Ad}\left(x_{1} \cdots x_{n-1}\right)\left(1-\operatorname{Ad}\left(x_{n}\right)\right)(\mathfrak{g}) .
\end{aligned}
$$

As

$$
L_{n}(x)=\left(1-\operatorname{Ad}\left(x_{1}\right)\right)(\mathfrak{g})+\operatorname{Ad}\left(x_{1}\right) L_{n-1}\left(x_{2}, \ldots, x_{n}\right),
$$

we have $\operatorname{dim} L_{n}\left(x_{1}, \ldots, x_{n}\right) \geq \operatorname{dim} L_{n-1}\left(x_{2}, \ldots, x_{n}\right)$ with equality if and only if

$$
\left(\operatorname{Ad}\left(x_{1}^{-1}\right)-1\right)(\mathfrak{g}) \subset L_{n-1}\left(x_{2}, \ldots, x_{n}\right) .
$$

Notice that $S=\operatorname{span}_{c \in C}\left(\operatorname{Ad}\left(c^{-1}\right)-1\right)(\mathfrak{g})$ is invariant under the adjoint action of $G$. As $c$ is not in the center of $G, S \neq 0$. Since the adjoint representation of $G$ is irreducible, $S=\mathfrak{g}$. Hence we can always increase the dimension of $L_{n+1}$, by prepending a word in $C^{n}$ by a suitable element in $C$, unless the dimension of $L_{n}$ is already $\operatorname{dim}(\mathfrak{g})$. Consequently, for each $x_{1} \in C$ there exist points $x_{2}, \ldots, x_{n} \in C$, $n=\operatorname{dim} G$ so that $u_{n}$ is submersive at $(e, \ldots, e)$. Lemma 3.3, specialized to $M=$ $N=G^{n}, P=G, m_{0}=(e, \ldots, e), n_{0}=\left(x_{1}, x_{2}, \ldots, x_{n}\right), p_{0}=e$, and the smooth map $W_{n}: G^{n} \times G^{n} \rightarrow G$ given by

$$
W_{n}\left(g_{1}, \ldots, g_{n}, y_{1}, y_{2}, \ldots, y_{n}\right)=g_{1} y_{1} g_{1}^{-1} g_{2} y_{2} g_{2}^{-1} \cdots g_{n} y_{n} g_{n}^{-1} y_{n}^{-1} \cdots y^{-1}
$$

produces neighborhoods $U_{i}$ of $x_{i}, 1 \leq i \leq n$, and an open neighborhood $V_{e}$ of $e$, for which

$$
V_{e} \subset W_{n}\left(G^{n}, y_{1}, y_{2}, \ldots, y_{n}\right),
$$

for all $y_{i} \in U_{i}$. As $x_{1}, x_{2}, \ldots, x_{n} \in C$, there exist $h_{2}, \ldots, h_{n} \in G$ such that $x_{i}=$ $h_{i} x_{1} h_{i}^{-1}$, and since the conjugation action of $G$ on $C$ is a smooth and invertible, some neighborhood $V$ of $x_{1}$ satisfies $V \subset U$ and $h_{i} V h_{i}^{-1} \subset U_{i}, 2 \leq i \leq n$. Thus, for any conjugacy class $C^{\prime}$ represented in $V$, there are $n$ elements $y_{1}, y_{2}, \ldots, y_{n}$ from $C^{\prime}$ for which

$$
V_{e} \subset\left(C^{\prime}\right)^{n} y_{n}^{-1} \cdots y_{2}^{-1} y_{1}^{-1} .
$$

By compactness of $G$, there exists $m \in \mathbb{N}$ such that $V_{e}^{m}=G$, so that

$$
G=\left(\left(C^{\prime}\right)^{n} y_{n}^{-1} \cdots y_{2}^{-1} y_{1}^{-1}\right)^{m}
$$

Since $y C^{\prime}=C^{\prime} y, y G=G$ for all $y \in G,\left(C^{\prime}\right)^{m n}=G$. Repeating the above construction for each $x$ in the compact set $G \backslash U_{e}$ and taking the maximum necessary exponent over a finite subcover proves the Theorem.

Remark 2. The argument for submersivity of the word map $u_{n}\left(g_{1}, \ldots, g_{n}\right)$ at $(e, \ldots, e)$ is similar to an argument in the proof of Proposition 3.2 of [1], where it was used to prove smoothness of $u_{n}$ for a larger class of groups.

Remark 3. Optimizing the smallest exponent in Theorem 1.2 is desired, but not necessary to proceed.

Remark 4 . If $G$ is compact, connected, simple but not adjoint simple, then Theorem 1.2 is false. Consider a conjugacy class $x C, x \in Z(G), C=\exp (\operatorname{Ad}(G) X), X \in \mathfrak{g}$. $Z(G)$ has finite order, say $m[4$. For any positive integer $n$, one can find a pair $X, k$ where $X$ is sufficiently near 0 in $\mathfrak{g}, k \in \mathbb{N}$ greater than $n$ but coprime to $|x|$ 
where $(x C)^{k}$ is contained in an open neighborhood around some element of $Z(G)$ that does not intersect $e$.

Remark 5. Products of conjugacy classes $C_{1}, C_{2} \cdots, C_{k}$ ought to be suitable for investigation using the methods of Theorems 1.2 and 2.9 .

\section{Character Estimates}

Lemma 4.1. Let $G$ be adjoint simple, $A$ a closed arc of the unit circle in $\mathbb{C}$. If $1 \notin A$, then there exists $\epsilon>0$ that satisfies the following property: For all characters $\chi$ of finite degree and all $g \in G$, if

$$
\frac{\chi(g)}{\chi(e)}=(1-\delta) \omega
$$

and $\omega \in A, 0 \leq \delta \leq 1$, then $\delta \geq \epsilon$.

Proof. Let $G$ be adjoint simple. By Theorem 1.2, there exists a bound $B>0$ such that $e \in C^{k}$ for all nontrivial conjugacy classes $C$ and all $k \geq B$. Let $A$ be a closed arc of the circle with $1 \notin A$. Treating $A$ as a closed interval in $(0,1)$, equip $\mathbb{R} / \mathbb{Z}$ with the distance induced from the nearest integer norm $\|x\|:=\lfloor x+1 / 2\rfloor$ on $\mathbb{R}$. Let $m \in[0,1 / 2]$ be the distance from $A+\mathbb{Z}$ to $\mathbb{Z}$. As $A$ is a closed interval and $(A+\mathbb{Z}) \cap \mathbb{Z}$ is empty, $m>0$. Pick $q \in \mathbb{N}$ to satisfy $1 / q<m$ and $q>B$. Obviously, $q \geq 2$, so there exists $\delta>0$ such that every $x$ within $\delta$ of the set

$$
S=\{t / s, 1 \leq t<s \leq q\},
$$

satisfies $j x \bmod 1 \in[1 / 4,3 / 4]$ for some $q \leq j \leq 2 q$. Now let $p \in \mathbb{N}$ satisfy $1 / p<\delta$, and let $x \in A$ be arbitrary. Consider the sequence

$$
x, 2 x, \ldots,(q+1) x \bmod 1 .
$$

Partitioning $[0,1]$ into $q$ intervals of length $1 / q$, by the pigeonhole principle, some pair of points lie in the same interval. Subtracting, we see that there is some integer $k$ between 1 and $q$ for which $\|k x\| \leq 1 / q$, i.e., $k x$ is within distance $1 / q$ of its nearest integer. Recalling $1 / q<m$ and $x \in A$ shows $k=1$ is impossible. Similarly, some term of the sequence

$$
k x, 2 k x, \ldots p k N \bmod 1
$$

satisfies $\|L k x\| \leq 1 / p$. As $1 / p<\delta,|L k x-n|<\delta$ for some $n \in \mathbb{Z}$, or

$$
|L x-n / k|<\delta / k<\delta .
$$

Thus, for every point $x$ in $A$, some term in the sequence

$$
x, 2 x, \ldots,(p+1) x \bmod 1
$$

lies within $\delta$ of $S+\mathbb{Z}$. Thus, if $\omega=e^{2 \pi i x}$ lies in $A$, then $\operatorname{Re} \omega^{k} \leq 0$ for some $k$ between $B$ and $2 p q$. We claim that $\epsilon=1 /(2 p q)^{2}$ satisfies the statement of the theorem. To this end, let $(\pi, V)$ be any finite dimensional representation of $G$, $g \in G$ arbitrary. Write $\chi=\operatorname{tr} \pi$ for the character of $\pi, C$ for the conjugacy class containing $y$ and $n=\operatorname{dim} V=\chi(e)$. Suppose

$$
\chi(g)=n(1-\delta) \omega
$$

where $\omega \in A, 1 \geq \delta \geq 0$. As $\omega \in A, g \neq e$. By Theorem 1.2 and the above derivation, there exists an integer $k, B \leq k \leq 2 p q$ and points $y_{1}, y_{2}, \ldots, y_{k} \in C$ that satisfy

$$
y_{1} \cdots y_{k}=e
$$


and $\operatorname{Re} \omega^{k} \leq 0$. In particular,

$$
\left|\omega^{k}-1\right| \geq \sqrt{2} .
$$

Write $P_{i}=\pi\left(y_{i}\right)$; each $P_{i}$ is unitary and any $P_{i}, P_{j}$ are unitarily equivalent. If $\lambda_{1}, \ldots, \lambda_{n}$ are the eigenvalues of $P_{i}$ then

$$
\lambda_{1}+\cdots+\lambda_{n}=n \omega(1-\delta),
$$

where $\omega$ and the $\lambda_{i}$ lie on the unit circle, so that

$$
\sum_{i=1}^{n}\left|\omega-\lambda_{i}\right|=\sum_{i=1}^{n}\left(2-\bar{\omega} \lambda_{i}+\omega \overline{\lambda_{i}}\right)=2 n \delta .
$$

Consider the Hermitian form $\operatorname{tr} A^{*} B$ on $M_{n}(\mathbb{C})$ which induces the so-called Frobenius norm

$$
\|A\|:=\sqrt{\operatorname{tr}\left(A^{*} A\right)}
$$

This norm satisfies the triangle inequality and is unitarily invariant, i.e., $\|U A\|=$ $\|A U\|=\|A\|$ for all $U \in U_{n}(\mathbb{C})$. Diagonalizing $P_{i}$ shows, by equation (4.5), that

$$
\left\|P_{i}-\omega I\right\|=\sqrt{2 n \delta}
$$

for each $P_{i}$. Writing $P_{1} \cdots P_{k}-\omega I$ as the telescoping sum

$$
P_{1} \cdots P_{k}-\omega P_{1} \cdots P_{k-1}+\omega P_{1} \cdots P_{k-1}+\cdots-\omega^{k-1} P_{1}+\omega^{k-1} P_{1}-\omega^{k} I,
$$

we get

$$
\begin{aligned}
& \left\|P_{1} \cdots P_{k}-\omega^{k} I\right\| \\
= & \left\|P_{1} \cdots P_{k}-\omega P_{1} \cdots P_{k-1}+\omega P_{1} \cdots P_{k-1}+\cdots-\omega^{k-1} P_{1}+\omega^{k-1} P_{1}-\omega^{k} I\right\| \\
\leq & \left\|P_{1} \cdots P_{k}-\omega P_{1} \cdots P_{k-1}\right\|+\cdots+\left\|\omega^{k-1} P_{1}-\omega^{k} I\right\| \\
= & \sum_{i=1}^{k}\left\|P_{i}-\omega I\right\|
\end{aligned}
$$

or $\left\|P_{1} \cdots P_{k}-\omega^{k} I\right\| \leq k \sqrt{2 n \delta}$. By equation (4.3),

$$
P_{1} \cdots P_{k}=I \text {. }
$$

and by estimate (4.4),

$$
\sqrt{2 n} \leq \sqrt{n}\left|\omega^{k}-1\right|=\left\|P_{1} \cdots P_{k}-\omega^{k} I\right\|,
$$

whence $1 / k^{2} \leq \delta$. As $k \leq 2 p q, \delta \geq 1 /(2 p q)^{2}=\epsilon$.

We are now ready to prove the main result.

Proof of Theorem 1.1. For $0<c<1$, let $\Theta_{c} \subset \mathbb{C}$ be the disk of radius $1-c$ centered at $c+0 i$. Proceeding by contradiction, suppose that for every $c$, there is a pair $x, \chi$ consisting of an element $x \in G$ and a character $\chi$ of $G$, such that $\chi(x) / \chi(e)$ lies outside $\Theta_{c}$. Any positive sequence $c_{1}, c_{2} \ldots$ that tends to 0 generates a list of complex numbers $z_{1}, z_{2}, \ldots$ coming from normalized character values, such that $z_{j}$ lies in the crescent

$$
\Theta_{0} \backslash \Theta_{c_{j}}=\left\{z \in \mathbb{C}|| z \mid \leq 1, z \notin \Theta_{c_{j}}\right\} .
$$

Such a sequence satisfies $z_{j} \neq 1 \forall j$ but $\left|z_{j}\right| \rightarrow 1$. By compactness, some subsequence of $\left\{z_{j}\right\}$ converges to a point on the unit circle; if this point is not equal to 1 , then the limit point is contained in a closed arc of $S^{1}$ not containing 1, contradicting Lemma 
4.1. So, the only possibility is $z_{j} \rightarrow 1$. Writing $z_{j}$ as $r_{k} e^{i \theta_{j}}$ where $-\pi \leq \theta_{j}<\pi$, then $z_{j} \rightarrow 1$ implies $\theta_{j} \rightarrow 0$ and $z_{j} \in \Theta_{0} \backslash \Theta_{c_{j}}$ means $\theta_{j} \neq 0$. Now, fix $0<c<1$ and parametrize the circular boundary of $\Theta_{c}$ as

$$
\Theta_{c}(\theta)=(1-c) \cos \theta+c+i(1-c) \sin \theta
$$

so that

$$
\left|\Theta_{c}(\theta)\right|=\sqrt{1-2 c(1-c)(1-\cos \theta)} .
$$

The estimate $\cos x \geq 1-x^{2} / 2$ yields

$$
\left|\Theta_{c}(\theta)\right| \geq \sqrt{1-c(1-c) \theta^{2}} \geq 1-c(1-c) \theta^{2} .
$$

for $\theta$ sufficiently small. In lemma 4.1 it was proved that if $z=\chi(g) / \chi(1)$ satisfies $\operatorname{Re} z^{k} \leq 0$ for some $k>B$ (B the bound of Theorem [1.2), then $|z| \leq 1-1 / k^{2}$. Since $\theta_{j} \rightarrow 0$ and $\theta_{j} \neq 0$ there exists $k>B$ and angle $\theta_{j}$ of $z_{j}$ for which

$$
\frac{\pi}{2(k+1)} \leq\left|\theta_{j}\right|<\frac{\pi}{2 k}
$$

So that $\left|z_{j}\right| \leq 1-1 / k^{2}$. By assumption, $\left|z_{j}\right|>\left|\Theta_{c_{j}}\left(\theta_{j}\right)\right|$ and the estimates (4.7), (4.8) yield the comparison

or

$$
1-\frac{1}{k^{2}}>1-c_{j}\left(1-c_{j}\right)\left(\frac{\pi}{2 k}\right)^{2},
$$

$$
\frac{4}{\pi^{2}}<c_{j}\left(1-c_{j}\right)
$$

contradicting $c(1-c) \leq 1 / 4$ for all $0<c<1$.

\section{REFERENCES}

[1] M. Larsen and A. Shalev. Word maps and Waring-type problems. J. Amer. Math. Soc., 2009.

[2] T. Rockafellar. Convex Analysis. Princeton University Press, 1970.

[3] V. Varadarajan. Lie Groups, Lie Algebras, and their Representations. Prentice Hall, 1974.

[4] W.Fulton and J.Harris. Representation Theory: A first course. Springer-Verlang, 1991.

Department of Mathematics, Amherst College, Amherst, MA 01002 